\title{
Pemphigus Foliaceous in a Dog - Clinical and Laboratorial Assessment
}

\author{
Virgínia Pereira Monteiro', Alexandre Tavares Camelo Oliveira² \& Tiago Cunha Ferreira²
}

\begin{abstract}
Background: The pemphigus complex is defined as a group of blistering autoimmune diseases that affects skin and mucous membrane. Pemphigus foliaceous is the most common disease in this group, being characterized by the production of autoantibodies against keratinocyte adhesion molecules. The treatment is based on the use of immunosuppressive drugs and requires constant monitoring to assess inflammatory control as well as side effects of therapy. Based on that, the aim of this study was to report the clinical and laboratorial follow-up of a canine with pemphigus foliaceous.

Case: An 11-year-old male neutered mongrel dog, weighing $9.8 \mathrm{~kg}$, was presented with a main complaint related to disseminated pruritus and lesions in face, trunk and limbs. Dermatological examination revealed meliceric crusts, epidermal collars and diffuse pustules in inguinal, abdominal, face, limbs, ears and thoraco-lumbar regions. Cytological examination was performed, revealing inflammatory and acantholytic cells and absence of bacterial cells. Biopsy procedure revealed subcorneal pustule with presence of epithelial acantholytic cells and neutrophils, compatible with canine pemphigus foliaceous. Hemato-biochemical analysis revealed a leukocytosis due to increased neutrophil count. Systemic treatment with high dose of prednisolone $(2.0 \mathrm{mg} / \mathrm{kg})$ and azathioprine $(2.5 \mathrm{mg} / \mathrm{kg})$ was proposed, while topical therapy with a $3 \%$ chlorhexidine shampoo was used to prevent secondary bacterial infections. The patient improved the dermatological clinical signs, being possible to observe a reduction of systemic and tissue inflammatory process. After 90 days of therapy, there was a partial loss of hair body coverage, associated with follicular lesions, and after 180 days of therapy it was possible to notice a new hair mantle, without visible areas of inflammation.

Discussion: The described clinical case demonstrates the clinical and laboratorial follow-up of a patient with naturally occurring canine pemphigus foliaceus. The main clinical characteristic of this disease is the formation of generalized pustular lesions, affecting regions such as the head, ear pinnae and limbs, which induced the diagnostic suspicion in the reported patient, in addition to the clinical history associated with complementary exams. In tissue evaluation, a pyogranulomatous inflammatory process was observed, with a marked presence of neutrophils and macrophages, which migrate from the dermis towards the epidermis. In systemic leukocyte analysis, an increase in the total leukocyte count was observed, due to the increase in circulating neutrophils. The therapy was based in use of a high dose of prednisolone associated with azathioprine. The following hemato-biochemical evaluations revealed a gradual reduction of systemic inflammatory process. Attention is drawn to neutrophil to lymphocyte ratio, which is a low-cost biomarker and less sensitive to pathophysiological changes when compared to individual leukocyte count. This parameter has been gaining visibility as a potential method of monitoring chronic inflammatory diseases. In veterinary medicine, its use is limited, with no reports related to canine pemphigus foliaceous in Brazil. After clinical and hematological improvement, prednisolone dose was slowly reduced, in order to avoid side effects. After clinical improvement, only azathioprine was maintained, in order to prevent critical flares. This report provides a clinical and laboratorial follow-up of a canine with pemphigus foliaceous, as well as it is the first one to describe the use of neutrophil to lymphocyte ratio to monitor therapeutic progression. New studies and reports involving this biomarker in autoimmune diseases monitoring are encouraged.
\end{abstract}

Keywords: canine autoimmune disease, neutrophil to lymphocyte ratio, immunosupressive drugs. 


\section{INTRODUCTION}

The pemphigus complex is defined as a group of autoimmune diseases that affect the skin and mucous membranes of patients, causing the formation of superficial or deep blisters [17]. Among this group, pemphigus foliaceus (PF) is mentioned as the most common autoimmune skin disease in the veterinary clinical routine [5].

Although it does not have a well-established etiology, it is known that immunopathogenesis of PF involves the naturally or induced production of autoantibodies against keratinocyte adhesion molecules, specifically desmoglein and desmocollin $[1,3]$. The binding of antibodies to such molecules promotes the breakdown of intercellular cohesion, culminating in superficial pustules with inflammatory and acantholytic cells (Figure 1) $[1,4]$.

Based on its inflammation chronicity, the therapies aim to attenuate the activation of the immune response and, consequently, reduce skin damage $[2,6]$. Conventional drugs, however, are not exempt from side effects, being potentially harmful to different biological systems due to the duration of the drug protocols $[4,6]$. Therefore, the treatment of PF requires constant monitoring to assess the control of the systemic and tegumentary inflammatory response, in addition to maintaining organic integrity.

Considering the chronicity of PF in dogs and the low number of reports of this disease, the objective of the

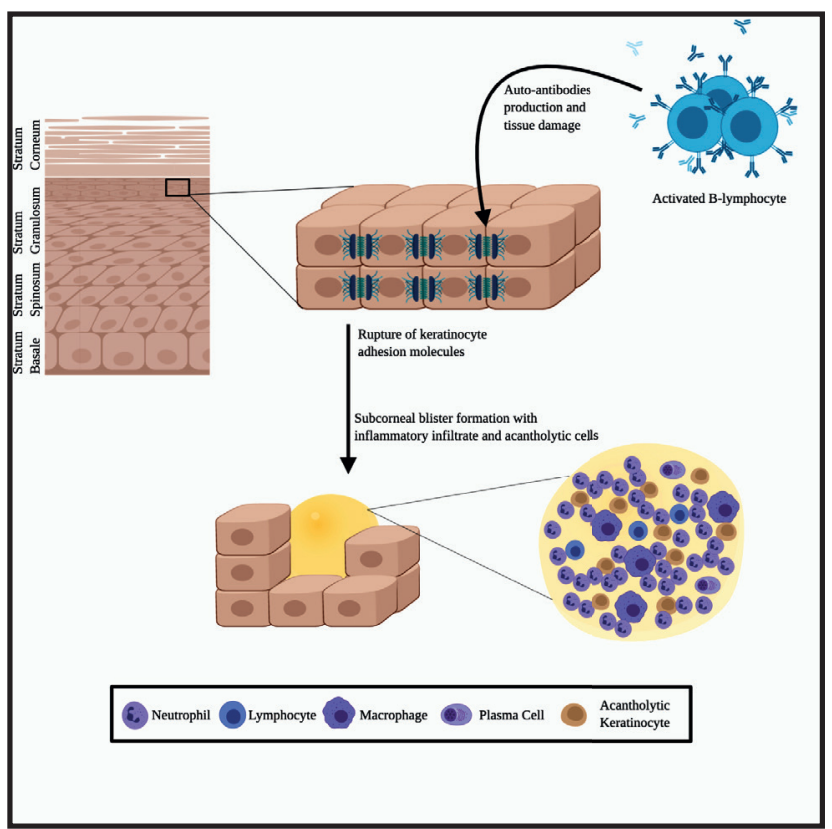

Figure 1. Immunopathogenic mechanism involved in canine pemphigus foliaceous.

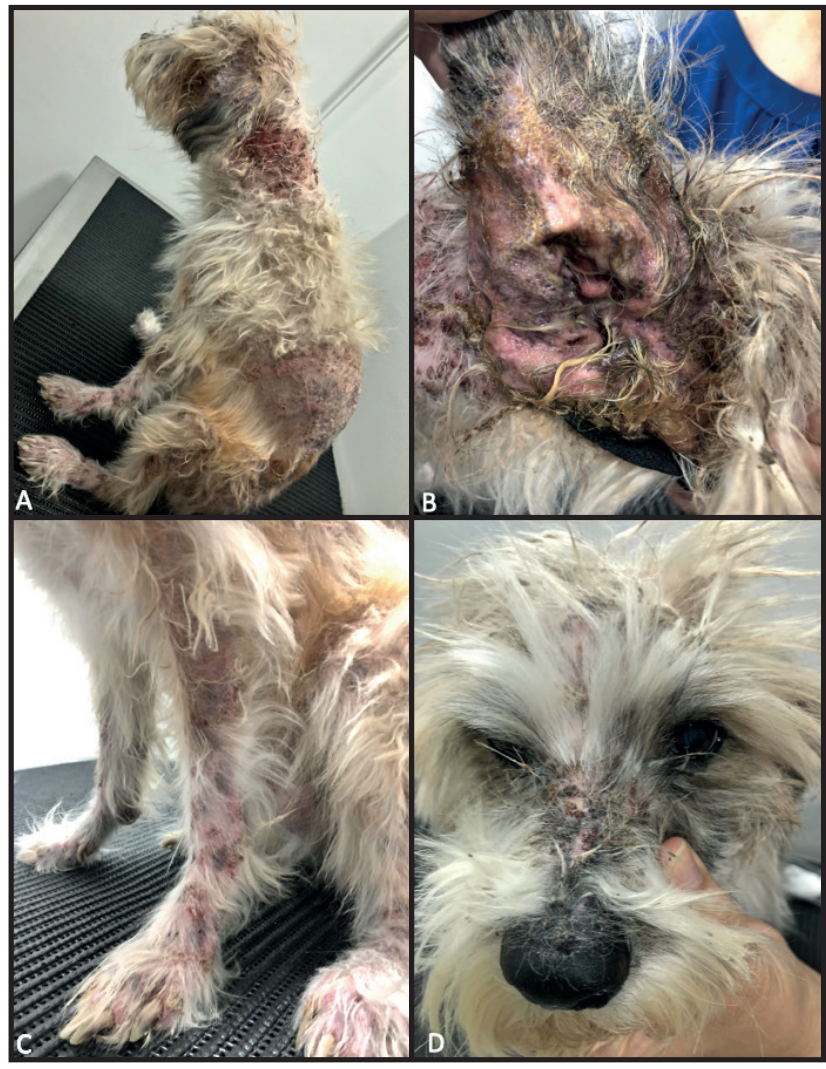

Figure 2. Canine patient affected by Pemphigus Foliaceous. A- Genera condition of the patient with alopecic areas, erythema, abrasions and diffuse hemato-meliceric crusts. B- Ear pinnae with the presence of erythema and purulent secretion. C- \& D- Affection of limbs and face with crusty, erythematous and purulent lesions.

present study is to describe the clinical and laboratorial follow-up of a canine with pemphigus foliaceus.

\section{CASE}

An 11-year-old male neutered mongrel dog, weighing $9.8 \mathrm{~kg}$, was presented with a history of disseminated blistering lesions, with purulent content and the presence of pruritus with evolution of 4 months. The patient had been previously diagnosed with atopic dermatitis, however he was unresponsive to antibiotics and steroid therapy.

Upon dermatological physical examination, the patient had diffuse erythema, pustules and hemo-meliceric crusts, affecting the back and belly, as well as the face, ears and limbs (Figure 2). Upon general clinical examination, the animal was active, temperature $39.2^{\circ} \mathrm{C}$, hydrated and presented reactive submandibular lymph nodes. On the occasion, blood sample was collected for hemato-biochemical and serological exams for Leishmaniasis, pustular secretion was drained for cytological evaluation and cutaneous biopsy of intact pustule was performed for histopathological analysis. 


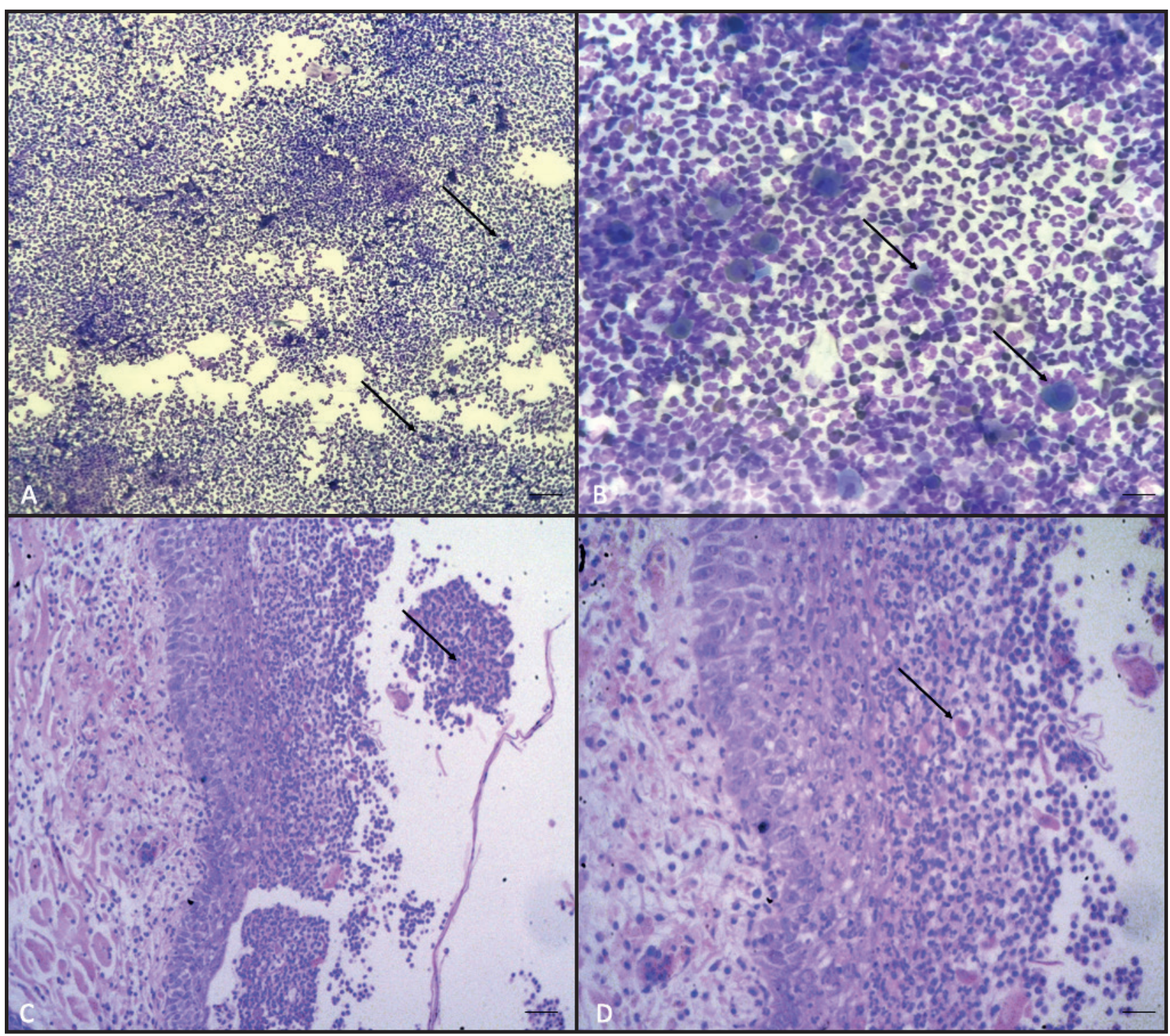

Figure 3. Cytological and histopathological aspects of pustule in a canine patient with pemphigus foliaceus. A- \& B- Cytology of pustular secretion with high presence of acantholytic keratinocytes (arrow), surrounded by intact neutrophils and macrophages, characterizing the Tzanck figure (Original magnification: A- 100x \& B- 400x). C- \& D-Histopathological evaluation of pustule, with subcorneal bullous formation with the presence of acantholytic keratinocytes (arrow), neutrophils and macrophages (Original magnification: C- 100x \& D- 400x) [H\&E; bar= $50 \mu \mathrm{m}]$.

The hematological evaluation revealed mild anemia and intense leukocytosis, due to neutrophilia, in addition to a high neutrophil/lymphocyte ratio (NLR). The serum levels of creatinine and alanine aminotransferase (ALT) were within the normal range. The animal was seronegative for Leishmaniasis.

Regarding the cytological findings, it was possible to observe a pyogranulomatous inflammatory process with a high density of acantholytic keratinocytes (Figure 3), in addition to the absence of bacterial cells. In histopathological analysis, a subcorneal pustule was seen, composed of neutrophils, macrophages, lymphocytes and acantholytic keratinocytes. When taken together, these findings suggest the diagnosis of canine pemphigus foliaceous.

Based on the results of the exams, therapy with Imuran $^{\circledR 1}[2.5 \mathrm{mg} / \mathrm{kg} / \mathrm{SID}$ of azathioprine $]$ and Alcort ${ }^{\circledR 2}$ [2.5 $\mathrm{mg} / \mathrm{kg} / \mathrm{SID}$ of prednisolone] was proposed in the induction process for treatment of autoimmune disease, in addition to baths with $3 \%$ chlorhexidine shampoo $\left(\text { Hexadene }^{\circledR}\right)^{3}$ in order to avoid secondary bacterial infections. It was also recommended to perform sequential and paired hematological and biochemical tests, due to the possible side effects associated with the instituted therapy. In these tests, it was possible to observe a gradual reduction in the values of total leukocytes, neutrophils, lymphocytes 
Table 1. Timeline of laboratory test results of a canine patient with pemphigus foliaceus.

\begin{tabular}{|c|c|c|c|c|c|c|c|}
\hline \multirow{2}{*}{ Parameter } & \multicolumn{7}{|c|}{ Timeline } \\
\hline & D0 & D30 & D60 & D90 & D120 & D180 & Min-Max* \\
\hline $\mathrm{RBC}\left(\mathrm{x} 10^{6} / \mu \mathrm{L}\right)$ & 4.9 & 4.1 & 4.2 & 4.7 & 5.1 & 5.1 & $5.5-8.5$ \\
\hline Hematocrit (\%) & 35.4 & 31.5 & 26.9 & 32.8 & 34.7 & 35.1 & $37-55$ \\
\hline $\mathrm{WBC}\left(\mathrm{x} 10^{3} / \mu \mathrm{L}\right)$ & 41.1 & 23.9 & 18.3 & 15.7 & 11.1 & 9.7 & $6.0-17.0$ \\
\hline Neutrophils $\left(\times 10^{3} / \mu \mathrm{L}\right)$ & 33.1 & 17.4 & 13.7 & 12.6 & 8.5 & 7.0 & $3.0-11.5$ \\
\hline Lymphocytes $\left(\times 10^{3} / \mu \mathrm{L}\right)$ & 3.1 & 1.4 & 1.2 & 1.1 & 1.1 & 1.0 & $0.7-5.4$ \\
\hline Neu/Lym Ratio & 10.6 & 12.4 & 11.4 & 11.4 & 7.7 & 7.0 & - \\
\hline ALT/GPT (U/L) & 107 & 24 & 77 & 74 & 86 & 62 & $10.0-88.0$ \\
\hline Creatinine (U/L) & 1.5 & 0.9 & 0.9 & 0.8 & 1.0 & 0.9 & $0.5-1.5$ \\
\hline
\end{tabular}

*Reference [11].

and NLR (Table 1), as well as the maintenance of ALT and Creatinine values.

After the immunosuppressive therapy, there was a progressive remission of the skin inflammatory process, as well as a fall in the entire hair coverage previously present in the injured areas (Figure 4). At the point of lesion stabilization, a gradual reduction of steroid therapy and maintenance of monotherapy with azathioprine $^{1}[2.5 \mathrm{mg} / \mathrm{kg} / \mathrm{SID}]$ was proceeded. After 180 days of therapy (Figure 5), it was possible to observe a new hairy mantle, with partial coverage of the skin. The animal is being followed up, with bimonthly reassessments, and it has not yet been possible to proceed with the total removal of the immunosuppressive drug.

\section{DISCUSSION}

The described clinical case demonstrates the clinical and laboratorial follow-up of a patient with naturally occurring canine pemphigus foliaceus. This disease is part of the group of vesicular diseases, with a high prevalence when compared to other autoimmune diseases [5]. Its main clinical characteristic is the formation of generalized pustular lesions, affecting regions such as the head, ear pinnae and limbs [4], which induced the diagnostic suspicion in the reported patient, in addition to the clinical history associated with complementary exams.

Superficial pyoderma, which also courses with disseminated pustules and crusts, is mentioned as an important differential diagnosis of canine PF [7]. This disease is usually caused by microorganisms from the cutaneous microbiota and is commonly associated with allergic diseases [15]. Due to the previous history of atopic dermatitis, the possibility of bacterial skin disease was initially considered. However, the patient did not show a satisfactory response to antibiotic therapy, and it was not possible to see bacterial cells in a cytological screening test, taking ruling out the possibility of infection.

Pustular lesions in PF are caused by the rupture of intercellular adhesion molecules, through the production and deposition of autoantibodies against desmosomal proteins [1,3]. The tissue damage caused by this interaction culminates in superficial epidermal bubbles with purulent content, composed of acantholytic keratinocytes and inflammatory cells (Figure 1), which were observed in cytological and histopathological evaluations (Figure 3).

In tissue evaluation, a pyogranulomatous inflammatory process was observed, with a marked 


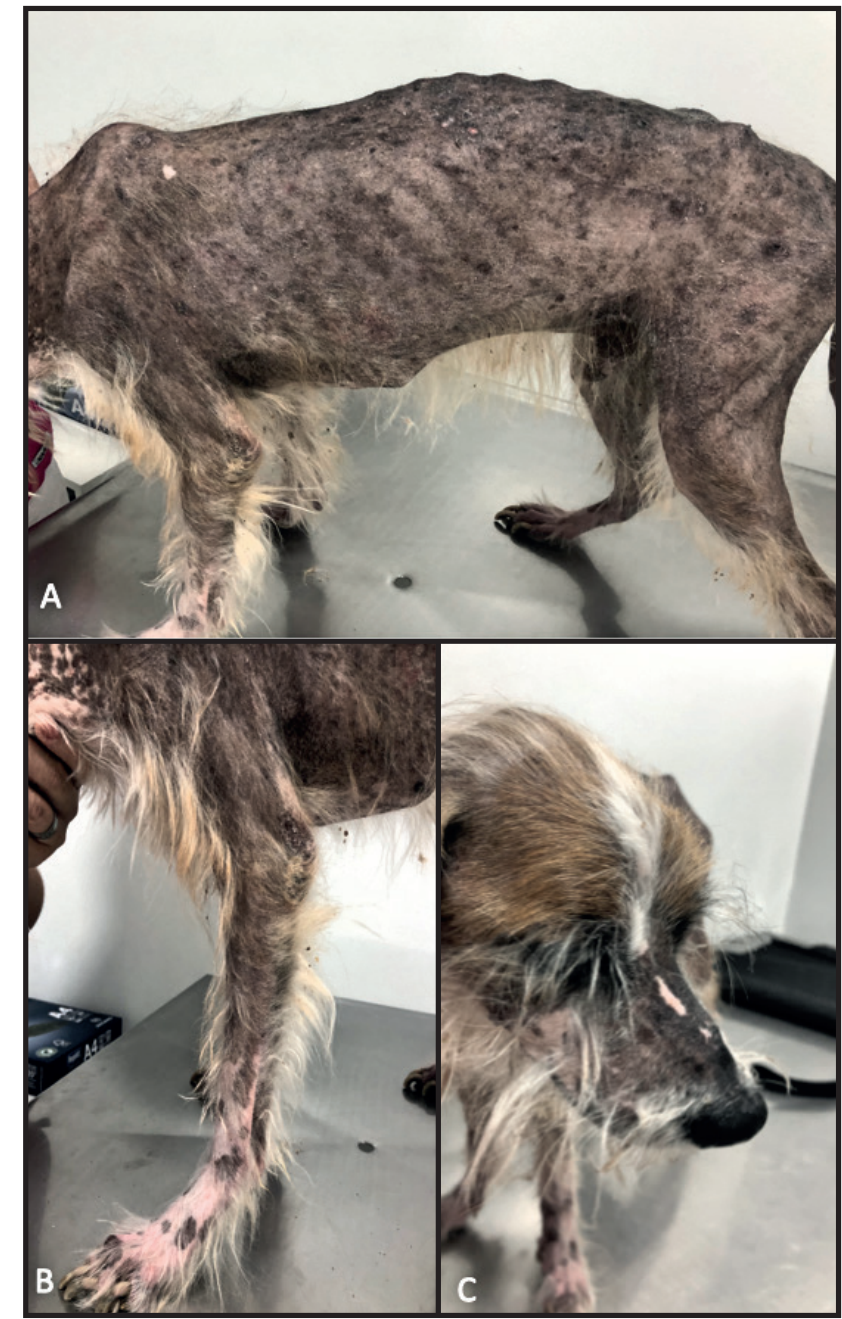

Figure 4. Clinical-dermatological aspects of the canine patient with pemphigus foliaceus, 80 days after the institution of immunosuppressive therapy. A- Hair fall, with foci of melanoderma and leukoderma, without areas of erythema. B \& C- Aspects of limbs and face, with evident loss of hair and reduction of crusty processes. It is still possible to observe areas of erythema and nail fragility.

presence of neutrophils and macrophages, which migrate from the dermis towards the epidermis. In systemic leukocyte analysis, an increase in the total leukocyte count was observed, due to the increase in circulating neutrophils (Table 1). These data represent the maintenance of an acute proinflammatory state in the patient, which may be related to the increase in tissue demand. Inflammation of the skin induces the production of chemotactic molecules and granulocyte colony stimulating factor (GCS-F), which, together with vascular adhesion molecules [12], contribute to the exit of neutrophils from the blood vessel in towards the affected tissue [14]. To control this inflammatory response, drugs that negatively modulate immune activation are used, such as prednisolone and azathioprine $[4,6]$. Steroid therapy, represented by prednisolone,

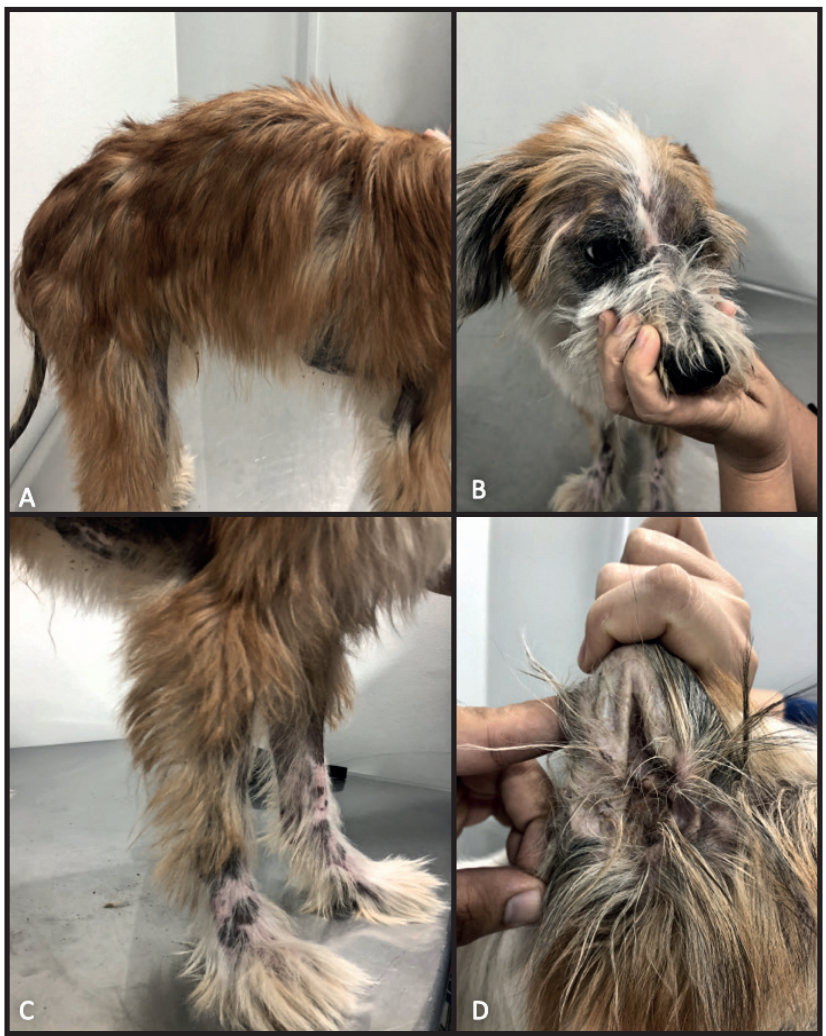

Figure 5. Clinical-dermatological aspects of a canine patient with pemphigus foliaceus after 180 days of immunosuppressive therapy. A-, B- \& C- A marked reduction is observed in the integumentary inflammatory process, as well as partial repilation in the face, trunk and limbs. D- A reduction in the auricular inflammatory process is observed, without the presence of erythema and purulent secretions.

is the basis for the treatment of PF, however, due to the side effects, systemic therapy with corticosteroid-sparing drugs is recommended [13]. Among them, azathioprine is mentioned, which acts by inhibiting cellular and humoral immune responses [10]. As side effects associated with the use of this medication, pancytopenia and hepatotoxicity can be observed [18], with constant hematological monitoring being recommended. In the reported patient, hemato-biochemical abnormalities associated with the use of azathioprine were not seen.

After the immunosuppressive therapy, it was possible to observe an improvement in the tissue inflammatory process, as well as a reduction in systemic leukocyte parameters (Table 1). Among these parameters, attention is drawn to the neutrophil/lymphocyte ratio, which is a laboratory biomarker that quantifies systemic inflammation, being used in human medicine to assess the prognosis in different types of neoplasms and inflammatory diseases $[8,16]$.

Within the group of autoimmune diseases, NLR has already been evaluated in patients with 
systemic lupus erythematosus, providing information about the inflammatory dynamics present in the disease [9]. Since it is a low-cost biomarker and less sensitive to pathophysiological changes when compared to individual leukocyte count [19], this parameter has been gaining visibility as a potential method of monitoring and prognosis in chronic inflammatory diseases. In veterinary medicine, its use is limited, with no data available on the importance of this biomarker in monitoring autoimmune diseases. To date, this is the first case report of PF with a description of the use of NLR to monitor therapeutic progression.

After clinical improvement, a gradual reduction in steroid therapy was started, in order to avoid side effects associated with the drug [6]. In addition, azathioprine dosage was reduced, aiming at hepato-medullary protection, since the patient was clinically stable. The remission of the immune-mediated disease has not been achieved so far, and it is possible to notice the recurrence of skin lesions, although of low intensity, when the dose of azathioprine is reduced.

Since canine pemphigus foliaceous is an autoimmune disease present in the canine dermatological routine, requiring attention from the veterinarian to identify the clinical signs associated with this disease. In addition, as it is a chronic disease, it requires continuous monitoring in order to avoid or delay flares. Based on that, studies involving inflammatory biomarkers, such as neutrophil/lymphocyte ratio, are recommended, in order to stablish its real potential to monitor inflammation control.

\section{MANUFACTURERS}

${ }^{1}$ Aspen Pharma Indústria Farmacêutica Ltda. Serra, ES, Brazil.

${ }^{2}$ Cepav Pharma Ltda. São Paulo, SP, Brazil.

${ }^{3}$ Virbac do Brasil Indústria e Comércio Ltda. São Paulo, SP, Brazil.

Declaration of interest. The authors declare no conflicts of interest. The authors were responsible for the content and writing of the paper.

\section{REFERENCES}

1 Bizikova P., Dean G.A., Hashimoto T. \& Olivry T. 2012. Cloning and establishment of canine desmocollin-1 as a major autoantigen in canine pemphigus foliaceous. Veterinary Immunology and Immunopathology. 149(3-4): 197-207.

2 Bizikova P. \& Olivry T. 2015. Oral glucocorticoid pulse theraphy for induction of treatment of canine pemphigus foliaceous - a comparative study. Veterinary Dermatology. 26: 354-e77.

3 Bizikova P., Olivry T., Mamo L.B. \& Dunston S.M. 2014. Serum autoantibody profiles of IgA, IgE and IgM in canine pemphigus foliaceus. Veterinary Dermatology. 25(5): 471-e75.

4 Ferreira T.C., Guedes R.F.M., Bezerra B.M.O. \& Nunes-Pinheiro D.C.S. 2019. Mupirocin Pemphigus-Like Drug Reaction in a Dog. Acta Scientiae Veterinariae. 47(Suppl 1): 429.

5 Gershwin L.J. 2010. Autoimmune diseases in small animals. Veterinary Clinics: Small Animal Practice. 40(3): 439-457.

6 Gomez S.M., Morris D.O., Rosenbaum M.R. \& Goldschmidt M.H. 2004. Outcome and complications associated with treatment of pemphigus foliaceus in dogs: 43 cases (1994-2000). Journal of the American Veterinary Medical Association. 224(8): 1312-1316.

7 Hillier A., Lloyd D.H., Scott-Weese J., Blondeau J.M., Boothe D., Breitschwerdt E., Guardabassi L., Papich M.G., Rankin S., Turnidge J.D. \& Sykes J.E. 2014. Guidelines for the diagnosis and antimicrobial therapy of canine superficial bacterial folliculitis (Antimicrobial Guidelines Working Group of the International Society for Companion Animal Infectious Diseases). Veterinary Dermatology. 25(3): 163-e43.

8 Krenn Pilko S., Langsenlehner U., Stojakovic T., Pichler M., Gerger A., Kapp K.S. \& Langsenlehner T. 2016. The elevated preoperative derived neutrophil-to-lymphocyte ratio predicts poor clinical outcome in breast cancer patients. Tumour Biology. 37: 361-368.

9 Ma L., Zeng A., Chen B., Chen Y. \& Zhou R. 2019. Neutrophil to lymphocyte ratio and platelet to lymphocyte ratio in patients with systemic lupus erythematosus and their correlation with activity: a meta-analysis. International Immunopharmacology. 76. DOI: 10.1016/j.intimp.2019.105949

10 Maltzman J.S. \& Koretzky G.A. 2003. Azathioprine: old drug, new actions. Journal of Clinical Investigation. 111(8): $1122-1124$.

11 Meyer D.J. \& Harvey J.W. 2004. Veterinary Laboratory Medicine: interpretation \& diagnosis. 2nd edn. Philadelphia: Saunders, 351p. 
12 Oh M.S., Hong J.Y., Kim M.N., Kwak E.J., Kim S.Y., Kim E.G., Lee K.E., Kim S.E., Jee H.M., Kim S.H., Sol I.S., Park C.O., Kim K.W. \& Sohn M.H. 2019. Activated Leukocyte Cell Adhesion Molecule Modulates Th2 Immune Response in Atopic Dermatitis. Allergy, Asthma \& Immunology Research. 11(5): 677-690.

13 Porro A.M., Hans Filho G. \& Santi C.G. 2019. Consensus on the treatment of autoimmune bullous dermatoses: pemphigus vulgaris and pemphigus foliaceus - Brazilian Society of Dermatology. Anais Brasileiros de Dermatologia. 94(2 Suppl 1): 20-32.

14 Sadik C.D., Kim N.D. \& Luster A.D. 2011. Neutrophils cascading their way to inflammation. Trends in Immunology. 32(10): 452-460.

15 Santoro D. \& Rodrigues Hoffmann A. 2016. Canine and Human Atopic Dermatitis: Two Faces of the Same HostMicrobe Interaction. Journal of Investigative Dermatology. 136: 1087-1089.

16 Shi G., Zhao J. \& Ming L. 2017. Clinical significance of peripheral blood neutrophil-lymphocyte ratio and plateletlymphocyte ratio in patients with asthma. Journal of Southern Medical University. 37(1): 84-88.

17 Tavakolpour S. 2017. Current and future treatment options for pemphigus: Is it time to move towards more effective treatments? International Immunopharmacology. 53:133-142.

18 Tavakolpour S. 2017. Pemphigus trigger factors: special focus on pemphigus vulgaris and pemphigus foliaceous. Archives of Dermatological Research. 310(2): 95-106.

19 Wang L., Wang C., Jia X., Yang M. \& Yu J. 2020. Relationship between Neutrophil-to-Lymphocyte Ratio and Systemic Lupus Erythematosus: a meta-analysis. Clinics. 75:e1450. DOI: 10.6061/clinics/2020/e1450 